\title{
DIE HARD HOLOGRAPHIC PHENOMENOLOGY OF CUPRATES
}

\author{
D.V. Khveshchenko \\ Department of Physics and Astronomy, University of North Carolina, Chapel Hill, NC 27599 \\ Email:khvesh@physics.unc.edu
}

Received 3 December 2020; accepted 6 January 2021

\begin{abstract}
We discuss the attempts of fitting a number of the approximate power-law dependences observed in the cuprates into one consistent holographic or holographically inspired hydrodynamic framework. Contrary to the expectations, the goal of reproducing as many as possible of the established behaviours of the thermodynamic and transport coefficients appears to be achievable within the picture of a non-degenerate fermion fluid with quadratic dispersion. While not immediately elucidating the essential physics of the cuprates, this observation suggests a possible reason for which the previous attempts towards that goal have so far remained inconclusive.
\end{abstract}

Keywords: high- $T_{c}$ cuprates, applied holography, transport, hydrodynamics

\section{Transport in cuprates}

The normal state of the cuprate superconductors has long remained a challenge defying many attempts of its theoretical understanding. In the continuing absence of a fully satisfactory microscopic description, a modest goal has been that of constructing a more or less successful phenomenological description capable of accounting for most of the observed transport properties.

Initially, the phenomenologies of the cuprates focused on the much publicized dichotomy between the robust power-law behaviours of the longitudinal conductivity observed in the optimally doped YBCO (and, to a lesser extent, LSCO) compounds

$$
\sigma \sim T^{\alpha}
$$

with $\alpha_{\exp }=-1$ and the Hall angle

$$
\tan \theta_{\mathrm{H}} \sim T^{\beta}
$$

manifesting the exponent $\beta_{\exp }=-2$.
In the early theoretical proposals, Eqs. (1, 2) were argued to imply the existence of two distinct scattering times, $\tau \sim T^{-1}$ and $\tau_{\mathrm{H}} \sim T^{-2}$, which were supposed to characterize the relaxation of either longitudinal vs transverse [1], charge-symmetric vs antisymmetric [2] currents, or a two-fluid nature of charge and heat transport [3]. Yet another insightful proposal of the 'marginal Fermi liquid' phenomenology was put forward early on [4].

Additional evidence of anomalous transport in the cuprates was provided by the magnetoresistivity

$$
\frac{\Delta \rho}{\rho} \sim T^{\gamma}
$$

that violates the conventional Kohler's law $\Delta \rho / \rho \sim B^{2} / \rho^{2}$, instead featuring the exponent $\gamma_{\exp }=-4$ [ [5] (in strong fields $B \gg T$ the quadratic field dependence changes to a linear one).

The anomalous transport properties (1-3) appear to coexist with the fairly conventional thermodynamic ones, including the Fermi-liquid-like specific heat and entropy 


$$
c \sim s \sim T^{v}
$$

with $v_{\text {exp }}=1$ [6]. In some compounds, upon approaching the pseudogap phase, $c(T)$ can also show a logarithmic enhancement, possibly signifying a quantum phase transition 何.

In the presence of thermal gradients, the combined thermo-electric response is described by the coefficients relating the charge $\mathbf{J}$ and heat $\mathbf{Q}$ currents to the gradients of electric potential and temperature,

$$
\begin{aligned}
& \mathbf{J}=\hat{\sigma} \mathbf{E}-\hat{\alpha} \nabla T, \\
& \mathbf{Q}=T \hat{\alpha} \mathbf{E}-\hat{\bar{\kappa}} \nabla T,
\end{aligned}
$$

where the $2 \times 2$ matrices such as, e.g. $\hat{\sigma}_{i j}=\sigma \delta_{i j}+\sigma_{\mathrm{H}} \epsilon_{i j}$, are composed of the longitudinal and transverse (Hall) components.

Early on the studies of heat transport focused on the Hall component as its longitudinal counterpart is believed to be dominated by the phonon contribution in most of the phase diagram [8, 9]. However, in the presence of chiral spin structures a sizable $\kappa_{\mathrm{H}}$ signal might also stem from phonons or magnons, or even both [10].

The list of the actually measured observables includes the thermopower (Seebeck) coefficient, thermal conductivities at zero current, the Hall Lorenz number, and Nernst coefficient

$$
\begin{aligned}
& S=\alpha / \sigma, \quad \kappa=\bar{\kappa}-\alpha^{2} / \sigma, \\
& L_{\mathrm{H}}=\kappa_{\mathrm{H}} / T \sigma_{\mathrm{H}}, \quad e_{\mathrm{N}}=\frac{\alpha_{\mathrm{H}} \sigma-\alpha \sigma_{\mathrm{H}}}{\sigma^{2}+\sigma_{\mathrm{H}}^{2}} .
\end{aligned}
$$

For some of these quantities the available data still remain scarce and their independent verification is badly needed. Nonetheless, the above coefficients (in the case of thermopower, its deviation from a possible constant term) might also exhibit the power-law dependences

$$
\begin{aligned}
& \kappa_{\mathrm{H}} \sim T^{\delta}, L_{\mathrm{H}} \sim T^{\lambda}, \\
& e_{\mathrm{N}} \sim T^{\mu}, S \sim T^{\rho},
\end{aligned}
$$

where $\rho_{\exp } \approx 1, \delta_{\text {exp }}, \mu_{\text {exp }}<0$, and $\lambda \geq 0$.

More specifically, in Refs. [8, 9] the data on $L_{\mathrm{H}}$ in the untwinned samples of optimally doped
$\mathrm{YBaCuO}$ were fitted into a linear dependence $(\lambda=1)$ while the Nernst signal $e_{\mathrm{N}}$ was found to increase dramatically with decreasing temperature. This effect was attributed to the superconducting fluctuations and/or disordered vortex pairs whose (positive) contribution dominates over that of the quasiparticles (whose sign, in turn, depends on the dominant type of carriers) upon approaching $T_{\mathrm{c}}$. Besides, $e_{\mathrm{N}}$ turned out to be strongly affected by a proximity to the pseudogap regime and can even become anisotropic [11-14].

However, the subsequent Refs. [15-17] reported somewhat different results for $\sigma_{\mathrm{H}}$ and $\kappa_{\mathrm{H}}$, and the concomitant slower temperature dependence of $L_{\mathrm{H}}$ in the $\mathrm{LaSrCuO}, \mathrm{EuBaCuO}$ and $\mathrm{YBaCuO}$ compounds. Specifically, in the twinned $\mathrm{YBaCuO}$ samples the measured exponents were

$$
\beta_{\exp }^{[15-17]}=-1.7, \delta_{\exp }^{[15-17]}=-1.2, \lambda_{\exp }^{[15-17]}=0.5 .
$$

Unlike Refs. [8, 9], the measurements in Refs. [1517] of both $\sigma_{\mathrm{H}}$ and $\kappa_{\mathrm{H}}$ were carried out on the same, rather than different, samples.

Adding to the puzzle of the cuprates' transport properties, there have been persistent reports of the Fermi liquid-like rate of inelastic quasiparticle scattering [18-22, 25]

$$
\Gamma_{\mathrm{qp}} \sim T^{2},
$$

in contrast to the almost uniformly accepted [23 and seemingly ubiquitous (see, however, Ref. [24]) 'Planckian' dissipation rate that is believed to control local equilibration/thermalization

$$
\Gamma_{\mathrm{eq}} \sim T .
$$

Generally, the latter would be expected in a quantum-critical phase associated with a quantum phase transition and in the absence of an intrinsic energy scale, other than temperature.

In the context of the cuprates, a number of the potentially viable quantum critical transitions have been discussed, their list including superconducting, spin, charge, nematic, as well as other, even more exotic, instabilities. However, some data [25] indicate that the quantum critical scenario may not necessarily be at work.

Nevertheless, the belief in the universality of Eq. (10) and its interpretation as a key evidence in 
support of the strong (as opposed to just moderate) correlations in the cuprates has brought to life a number of proposals based on the various 'ad hoc' generalizations of the original ground-breaking conjecture of holographic correspondence.

\section{Applied holography}

In its own words, the 'bottom-up' applied holography (a.k.a. AdS/CMT or anti-deSitter/condensed matter theory correspondence) purports to offer a unique, intrinsically strong-coupling, approach to a variety of the traditionally hard condensed matter problems [26-32]. On the technical side, this intriguing (albeit still lacking any solid proof) scheme borrows its computational apparatus (in essence, 'ad verbatim') from the original machinery of the conjectured holographic AdS/CFT (antideSitter/conformal field theory) correspondence which was developed and professed in the 'bona fide' string/field theory.

From the conceptual standpoint, searching for a common cause of the observed properties would indeed make perfect sense if the sought-after universality were indeed present. However, under a closer inspection even some close members of the same family of materials often demonstrate different behaviours and exhibit different power laws. Obviously, any significant diversity between the related compounds would be rather difficult to accommodate under the holographic paradigm, since virtually every compound would then require individual treatment and a material-specific dual bulk geometry.

Such potential difficulties notwithstanding, the decade-long vigorous work on the AdS/CMT exploited a variety of the classical geometries (Reissner-Nordström, Lifshitz, hyperscaling-violating, Bianchi, Q-lattices, etc.) [26-32]. Such exploratory studies resulted in a number of rather exotic proposals for obtaining some of the exponents (1-4, 7), although in order to reproduce even the basic Eqs. $(1,2)$ such analyses would often have to go to a great length.

To that end, one popular scheme [33] invokes the extreme 'ultra-local' $\mathrm{AdS}_{2}$ limit, where both the dynamical critical index $z$ and the 'hyperscaling-violation' exponent $\theta$ take infinite values, thereby conspiring to make the conductivity inversely proportional to the entropy density

$$
\sigma \sim T^{(\theta-2-z) / z} \sim \mathcal{s}^{-1} T^{-2 / z}
$$

in order to conform to Eq. (1) for $z \rightarrow \infty$.

Another, more comprehensive, attempt was made in Ref. [34] where the values $z=4 / 3$ and $\theta=0$ were argued to reproduce the observed behaviour of the transport coefficients $(1-3)$ while $\lambda_{\text {holo }}^{[34]}=1$ was chosen as one of the constitutive relations (despite the fact that, unlike Refs. [8, 9], the works of Refs. [11-17] reported a slower-than-linear in - or even decreasing with - $T$ electronic Lorenz ratio), yet still other exponents

$$
v_{\text {holo }}^{[34]}=1.5, \rho_{\text {holo }}^{[34]}=0.5, \mu_{\text {holo }}^{[34]}=-1.5
$$

were markedly off their targeted values (4), and also

$$
\rho_{\exp }=0 \text { or } 1 \text {, }
$$

depending on whether the goal was to fit the constant (which may or may not have been of electronic origin) or the linear term in the experimental plot for $\mathrm{LaSrCuO}$ [35]

$$
S=a-b T
$$

In that regard, the analysis of Ref. [34] ignored the constant and went straight for the $T$-dependent term.

For comparison, the alternate scaling scheme of Ref. [36] characterized by the least exotic values $z=\theta=1$ readily produces the exponents Eqs. (1-4), alongside

$$
v_{\text {holo }}^{[36]}=1, \rho_{\text {holo }}^{[36]}=1,
$$

and, to a lesser extent, $\lambda_{\text {holo }}^{[36]}=0$. All of the above appear to generally agree with experiment (there was not enough data available for ascertaining the predicted values of $\mu_{\text {holo }}^{[36]}=-1$ and $\delta_{\text {holo }}^{[36]}=-2$, though).

Furthermore, the results of Ref. [34] were argued to compare favourably with certain characteristics of the energy- and momentum-dependent magnetic susceptibility, as probed by inelastic neutron scattering in $\mathrm{LaSrCuO}$. Specifically, the predicted behaviour of the ratio (here $\mathbf{Q}$ is the antiferromagnetic vector)

$$
\left.\frac{\chi_{\mathrm{s}}(\omega, \mathbf{q})}{\omega}\right|_{\omega \rightarrow 0} \sim|\mathbf{q}-\mathbf{Q}|^{\eta}
$$


was found to be governed by the exponent $\eta_{\text {holo }}=-10 / 3$ which is indeed close to the measured $\eta_{\exp }=-3[37,38]$. In contrast, the alternate scheme of Ref. [36] yields the exponent $\eta=3$ the value of which appears to be right on the data.

Also, the momentum integral $\left.T \int \mathrm{d} \mathbf{q} \frac{\chi_{\mathrm{s}}(\omega, \mathbf{q})}{\omega}\right|_{\omega \rightarrow 0}$

turned out to be constant in both schemes of Refs. 34 ,

36, again in agreement with the data of Refs. [37, 38. In turn, the uniform magnetic and charge susceptibilities

$$
\chi_{\mathrm{s}}=\frac{\mathrm{d}^{2} f}{\mathrm{~d} B^{2}} \sim T^{\xi}, \chi_{\mathrm{c}}=\frac{\mathrm{d}^{2} f}{\mathrm{~d} \mu^{2}} \sim T^{\xi}
$$

were found to be governed by the exponents

$$
\xi_{\text {holo }}^{[34]}=-1.5, \xi_{\text {holo }}^{[34]}=0.5
$$

and

$$
\xi_{\text {holo }}^{[36]}=-2, \xi_{\text {holo }}^{[36]}=0
$$

in Refs. [34, 36], respectively, thus providing additional means of discriminating between the two scenaria. Notably, in either scheme the Wilson ratio $c / \chi_{c} T$ conformed to a constant, once again in accord with experiment.

Although a few of the subsequent publications [39, 40] acknowledged the better overall agreement with the predictions of Ref. [36], great many other papers pursued the various versions of the holographic approach, akin to Refs. [33, 34.

To that end, the practical AdS/CMT has been reinventing itself as advanced hydrodynamics of strongly coupled quantum matter. The renewed appreciation for and novel applications of such a wellestablished field as hydrodynamics (which, while suggesting some formal holographic connections, had long been discussed before the rise of holography) emerged out of the recent experimental discoveries of the electron hydrodynamic regime in mono- and bilayer graphene, $(\mathrm{Al}, \mathrm{Ga})$ As heterostructures, $\mathrm{PdCoO}_{2}$, herbertsmithite, etc. Among other things, it also resulted in a renewed interest in the anomalous transport in the cuprates.

\section{3. (Non)holographic hydrodynamics}

The intimate relation between classical gravity and hydrodynamics has long been known as a particu- lar take on the AdS/CFT referred to as 'fluid-gravity' correspondence [26-32]. The crux of the matter lies in the deep similarity between the asymptotic near-boundary behaviour of the Einstein equations for the bulk metric and the Navier-Stokes ones describing a dual boundary fluid in one lesser dimension. Albeit usually truncated and, therefore, approximate such relations can be systematically improved, thus enabling certain computational simplifications.

The magneto-hydrodynamic transport coefficients were first derived in the early work of Ref. [41] under the assumption of a (pseudo) relativistic kinematics of the charge and heat carriers. While the underlying hydrodynamic equations were mimicked after those of a quark-gluon plasma, they would also be considered applicable to the electron transport in graphene (the actual hydrodynamic equations describing monolayer graphene appear to be somewhat different due to the presence of an extra hydrodynamic 'imbalance' mode as well as the expressly non-relativistic nature of the Coulomb interaction, though [42-45]).

To the lowest order in the magnetic field the hydrodynamic results of Ref. [41] reduce to the relations

$$
\begin{aligned}
& \sigma \sim \sigma_{\mathrm{coh}}+\sigma_{\mathrm{in}}, \\
& \sigma_{\mathrm{H}} \sim B \sigma_{\mathrm{coh}}\left(\sigma_{\mathrm{coh}}+2 \sigma_{\mathrm{in}}\right) n^{-1}, \\
& \alpha \sim s \sigma_{\mathrm{coh}} n^{-1}, \\
& \alpha_{\mathrm{H}} \sim s B \sigma_{\mathrm{coh}}\left(\sigma_{\mathrm{coh}}+\sigma_{\mathrm{in}}\right) n^{-2}, \\
& \kappa \sim s^{2} T \sigma_{\mathrm{coh}} n^{-2}, \\
& \kappa_{\mathrm{H}} \sim B s^{2} T \sigma_{\mathrm{coh}}^{2} n^{-3} .
\end{aligned}
$$

Regarding these expressions the all-time important issue has been that of the intrinsically additive or 'inverse Matthiessen' structure of the kinetic coefficients 46, 477.

In particular, the hydrodynamic (as well as the alternate memory-matrix) calculations of the DC conductivity revealed its decomposition onto the generalized coherent ('Drude') contribution 48-57]

$$
\sigma_{\mathrm{coh}}=\frac{\chi_{J P}^{2}}{\chi_{P P} \Gamma_{\mathrm{mr}}}
$$


and its intrinsic 'incoherent' counterpart. In the relativistically invariant holographic context it was estimated as

$$
\sigma_{\mathrm{inc}}=\left(\frac{s T}{\epsilon+P}\right)^{2},
$$

where $s$ and $\epsilon+P$ are entropy and enthalpy densities, respectively [58].

The coherent term (21) is controlled by the momentum relaxation rate $\Gamma_{\mathrm{mr}}$ together with the momentum-momentum $\chi_{P P}$ and current-momentum $\chi_{I P}$ susceptibilities. The latter vanishes if the operator of electric current is orthogonal to that of momentum.

The coherent term receives contributions from all the sources of momentum relaxation (impurities, phonons, umklapp, boundary scattering, etc.). In turn, the second term provides for a finite conductivity in a neutral relativistic plasma in the absence of any external mechanism of momentum relaxation. Physically, it is due to the momentumconserving Coulomb drag between the opposite charge carriers.

Similar incoherent terms were argued to appear in the other thermo-electric coefficients, $\alpha_{\text {inc }}=-\mu \sigma_{\text {inc }} / T$ and $\bar{\kappa}_{\text {inc }}=\mu^{2} \sigma_{\text {inc }} / T$, which, however, cancel against each other in the zero-current coefficient $\kappa$. Recently, it was argued that similar terms must be introduced into the Hall components of the kinetic coefficients as well [59].

According to the popular scenario of Ref. [60], in the conjectured quantum-critical regime the incoherent contribution $\sigma_{\text {inc }}$ is supposed to dominate the $\mathrm{Ohm}$ conductivity, thus determining the exponent $\alpha$, while the Hall response would be controlled by $\sigma_{\text {coh }}$.

Elaborating further on this proposal, in Refs. [61, 62] the coherent and incoherent terms, alongside the carrier density $n$, were chosen to behave as

$$
\begin{gathered}
\sigma_{\text {coh }}^{[61,62]} \sim T^{-2}, \sigma_{\text {inc }}^{[61,62]} \sim T^{-1}, \\
n^{[61,62]} \sim T^{0}, \quad s^{[61,62]} \sim T,
\end{gathered}
$$

as if the fermion system was deep in the degenerate regime and had a well developed Fermi surface with a finite Fermi momentum $\sim n^{0.5}$.

Besides, the scenario of Refs. [61, 62] produced a list of other exponents

$$
\begin{gathered}
\gamma_{\text {hydro }}^{[61,62]}=-3, \mu_{\text {hydro }}^{[61,62]}=-1, \\
\lambda_{\text {hydro }}^{[61,62]}=1, \rho_{\text {hydro }}^{[61,62]}=0,
\end{gathered}
$$

that could be contrasted against the data $(3,4,7)$ as well (spoiler: with only a limited success).

Also, the assumptions (23) were made in Refs. [63-65] where yet another version of the holographic, the so-called DBI (Dirac-Born-Infeld), approach was utilized, thus resulting in the same ostensible match for the experimental Eqs. (1-3).

However, in reality the desired dependences (23) might be rather difficult to conform to. Specifically, for a temperature-independent density $n$ the low$T$ behaviour becomes non-relativistic and Eq. (22) yields $\sigma_{\text {inc }} \sim s^{2} T^{2}$. Instead of behaving as $T^{-1}$, as per Eq. (23), $\sigma_{\text {inc }}$ then vanishes with temperature as $T^{4}$ and, therefore, could hardly compete with $\sigma_{\text {coh }} \sim 1 / \Gamma_{\mathrm{mr}}$. Indeed, the rate $\Gamma_{\mathrm{mr}}$ either remains almost constant (impurity scattering) or even decreases with decreasing $T$ (phonons or Baber umklapp scattering). Either way, the assumed $T^{-1}$ behaviour does not readily occur.

In the opposite, high- $T$, limit Eq. (22) approaches a temperature-independent constant of order unity (or, rather, $e^{2} / h$ ). This would be typical for, e.g. (pseudo)relativistic $2+1$-dimensional fermions in mono-layer graphene which are governed by the unscreened 3-dimensional Coulomb interactions.

Interestingly enough, this behaviour would also be shared by the zero-density fermions with a quadratic dispersion, akin to that in (untwisted) bilayer graphene. In the latter case, the density of thermal excitations $n \sim T$ cancels against the inelastic Coulomb scattering rate (10), thus yielding an (approximate) constant [66-71].

However, it should be kept in mind that in the limit of a strongly T-dependent carrier density, the criterion of 'hydrodynamicity' $\left(\Gamma \gg \Gamma_{\mathrm{mr}}\right)$ further decouples from the assumed dominance of $\sigma_{\text {inc }}$ which would be, by and large, controlled by $\mathrm{n} /$ / in with the pertinent inelastic rate given by, e.g. Eq. (9) or (10). As a result, the range of parameters at which hydrodynamics is expected to work might be bounded at both low- and high-T.

Indeed, in the presence of competing sources of momentum relaxation such recognized hydrodynamic systems as monolayer and magic-angletwisted bi-layer graphene were predicted to manifest their fluid-like behaviour only within a relatively narrow window of temperatures where the disorder 
and phonon scattering mechanisms set the lower and upper bounds, respectively. On the other hand, in the untwisted bilayer graphene the hydrodynamic regime is not expected to be bounded from above [66-71].

In that regard, it is instructive to mention the recent work on the compound BSCO with a low$T_{c} \sim 10 \mathrm{~K}$ [72] which reported the different measured exponents

$$
\beta_{\exp }^{[72]}=-1.5, \delta_{\exp }^{[72]}=-3, \mu_{\exp }^{[72]}=-2.5 .
$$

The new values of $\delta$ and $\mu$ were extracted from the data taken in the narrow range of temperatures between 20 and 40 or $60 \mathrm{~K}$ (above which the Nernst signal changes sign), respectively. For comparison, the results of Ref. [10] for $\sigma_{\mathrm{H}}, \kappa_{\mathrm{H}}$ and $L_{\mathrm{H}}$ in underdoped YBCO were collected over a wider range of temperatures, yet the authors refrained from fitting them with any particular power laws.

Nonetheless, the concomitant theoretical analysis of Ref. [72] based on the hydrodynamic Eqs. (20) claimed to have been able to explain all of Eqs. $(1,4,25)$ by making the assumptions

$$
\begin{gathered}
\sigma_{\mathrm{coh}}^{[72]} \sim T^{0}, \sigma_{\mathrm{inc}}^{[72]} \sim T^{-1}, \\
n^{[72]} \sim T^{1.5}, s^{[72]} \sim T .
\end{gathered}
$$

Similar to the earlier proposals 60-65] electrical transport was still going to be dominated by $\sigma_{\text {inc }}$. However, this time around it was supposed to occur at low rather than high temperatures between 10 and $100 \mathrm{~K}$ (despite the fact that the must-have exponent (1) could be observed up to $700 \mathrm{~K}$ ).

Moreover, in Ref. [72] the main source of momentum relaxation was attributed to the non-quasi-particle transport through a charge density wave (CDW) [73-75] and an intricate cancellation between the different $T$-dependent factors in $\sigma_{\text {coh }}$ was required to achieve (26).

Lastly, by having made the above assumptions, Ref. [72] would also be forced into the less wanted predictions

$$
\rho_{\text {hydro }}^{[72]}=-1, \lambda_{\text {hydro }}^{[72]}=-1.5,
$$

which are not immediately supported by the data.

In fact, if the agreement asserted in Ref. [72] were indeed there, the assumed behaviour of the carrier density (26) would have appeared to be in conflict with the underlying assumption of the relativistic (that is, $z=1$ ) kinematics of carriers, as well as the conjectured scaling of entropy. Besides, it would also call for the inelastic scattering rate $\Gamma_{\text {in }} \sim n / \sigma_{\text {inc }} \sim T^{2.5}$ for which there seems to be no known microscopic mechanism.

Indeed, in a generic $d$-dimensional system with the dispersion $\epsilon \sim p^{z}$ the carrier density scales as $n \sim T^{d / z}$. Thus, taken at its face value Eq. (26) would have implied $z=4 / 3$ for $d=2$. Incidentally, this value of $z$ coincides with that proposed in Ref. [34], despite the fact that instead of the novel Eq. (25) Ref. [34] aimed at reproducing the 'orthodox' values of the exponents in Eqs. (1-4, 7).

In light of the lingering tension between the available data and all the aforementioned proposals, it is interesting to point out that the hydrodynamic formulas can still produce the exponents that are equal or close to the observed ones by adopting the scenario of Ref. [36].

Namely, under the minimal assumptions

$$
\sigma_{\text {hydro }} \sim T^{-1}, n_{\text {hydro }} \sim T,
$$

where no distinction is to be made between the 'Drude' and incoherent parts of the conductivity and barring any fine-tuned cancellations $a$ la Sondheimer, the hydrodynamic Eqs. (20) yield the following exponents:

$$
\begin{array}{r}
\alpha_{\text {hydro }}^{[36]}=-1, \beta_{\text {hydro }}^{[36]}=-2, \gamma_{\text {hydro }}^{[36]}=-4, \\
\delta_{\text {hydro }}^{[36]}=-2, v_{\text {hydro }}^{[36]}=1, \mu_{\text {hydro }}^{[36]}=-2, \\
\lambda_{\text {hydro }}^{[36]}=0, \rho_{\text {hydro }}^{[36]}=0 .
\end{array}
$$

The ansatz (28) describes, e.g. a system of non-degenerate two-dimensional fermions with a quadratic dispersion and generic scattering rate (10).

Of course, the simple schemes with $T$-dependent carrier density and a single scattering rate, including those with $n \sim T$, have been discussed since the early days of the high- $T_{c}$ era $[76,77]$. As regards the cuprates, the underdoped $\mathrm{YBaCuO}$ and $\mathrm{HgBaCuO}$ show the presence of small electron pockets, in contrast with the large hole-like Fermi surface which develops in the overdoped regime above the critical doping $p *$. This is consistent with the reports of a dramatic drop in the low-temperature carrier density (evaluated by the Hall number 
$n_{\mathrm{H}}$ ) from $n_{\mathrm{H}} \approx 1+p$ to $n_{\mathrm{H}} \approx p$ upon crossing into the pseudogap phase [10-14].

The generic rate (9) could originate from the Baber mechanism (although the applicability of hydrodynamics would then be rather questionable) whose effectiveness depends on whether or not the quasiparticle dispersion, Fermi surface topology, and spatial dimension conspire to provide for the comparable rates of the normal and umklapp inelastic scattering processes. It is believed, though, that in the cuprates both the multipocketed (in the under- and optimally-doped cases) as well as the extended concave (in the overdoped case) hole Fermi surfaces might comply with the necessary conditions outlined in Refs. [78-80].

The matters become further complicated due to the possible onset of CDW order, as in orthorhombic $\mathrm{YBCO}$ and tetragonal $\mathrm{HgBaCuO}$ which may induce Fermi-surface reconstruction. However, some authors believe that this might be a secondary phenomenon occurring only in high magnetic fields and at temperatures below the zero-field $T_{c}$ 25.

By contrast, when coupled with the Fermi liquid-like scattering rate (9) observed across much of the entire cuprates' phase diagram [18-22, 25, the $T$-dependent carrier density may be hinting at some alternate theoretical scenaria that neither exploit the notion of quantum criticality, nor attribute any special role to the incidental CDW order. In particular, the work of Ref. [25] emphasizes a potential importance of the local (pseudo)gaps produced by some intrinsic microscopic inhomogeneity (whose type of local physics would unlikely be conducive to any holographic speculations).

\section{Summary}

To summarize, this note exposes the systemic problem inherent to the 'bottom-up' holographic approach which tends to favour technical convenience at the expense of physical insight.

It is, of course, quite possible that the seemingly suggestive scaling exhibited by a variety of the experimental probes is, in fact, only approximate and limited to certain, insufficiently broad, ranges of parameters, thus making it virtually impossible to explain all such findings within the same paradigm.

Moreover, as regards the general holographic task of constructing a comprehensive catalogue of all the different types of 'strange-metallic' behaviour [26-32], the traditional focus on the cuprates with its prime goal of reproducing the above scaling dependences appears to be much too narrow. To that end, there exists a plethora of other (e.g. heavyfermion) compounds where the unexplained powerlaw dependences are abound. As an added challenge, in many cases the apparent exponent $z$ remains finite, thus breaking out of the restrictive confines of the $\mathrm{AdS}_{2}$ scenario characterized by the diverging $z$.

Thus, should the true status of the holographic studies be finally ascertained and its machinery proved viable, there would still be a plenty of other condensed matter systems [81] waiting to be tackled by this purportedly promising method for studying strongly correlated systems.

\section{References}

[1] P.W. Anderson, Hall effect in the two-dimensional Luttinger liquid, Phys. Rev. Lett. 67, 2092 (1991), https://doi.org/10.1103/PhysRevLett.67.2092

[2] P. Coleman, A.J. Schofield, and A.M. Tsvelik, Phenomenological transport equation for the cuprate metals, Phys. Rev. Lett. 76, 1324 (1996), https://doi.org/10.1103/PhysRevLett.76.1324

[3] D.K. Lee and P.A. Lee, Transport phenomenology for a holon-spinon fluid, J. Phys. Cond. Mat. 9, 10421 (1997), https://doi.org/10.1088/0953$8984 / 9 / 47 / 010$

[4] C.M. Varma, P.B. Littlewood, S. Schmitt-Rink, E. Abrahams, and A.E. Ruckenstein, Phenomenology of the normal state of $\mathrm{Cu}-\mathrm{O}$ hightemperature superconductors, Phys. Rev. Lett. 63, 1996 (1989), https://doi.org/10.1103/ PhysRevLett.63.1996

[5] J.M. Harris, Y.F. Yan, P. Matl, N.P. Ong, P.W. Anderson, T. Kimura, and K. Kitazawa, Violation of Kohler's rule in the normal-state magnetoresistance of $\mathrm{YBa}_{2} \mathrm{Cu}_{3} \mathrm{O}_{7-}$ and $\mathrm{La}_{2} \mathrm{Sr}_{x} \mathrm{CuO}_{4}$, Phys. Rev. Lett. 75, 1391 (1995), https://doi.org/10.1103/ PhysRevLett.75.588

[6] J.W. Loram, K.A. Mirza, J.M. Wade, J.R. Cooper, and W.Y. Liang, The electronic specific heat of cuprate superconductors, Physica C 235-240, 134 (1994), https://doi.org/10.1016/09214534(94)91331-5 
[7] B. Michon, C. Girod, S. Badoux, J. Kačmarčík, Q. Ma, M. Dragomir, H.A. Dabkowska, B.D. Gaulin, J.-S. Zhou, S. Pyon, et al., Thermodynamic signatures of quantum criticality in cuprate superconductors, Nature 567, 218 (2019), https:// doi.org/10.1038/s41586-019-0932-x

[8] Y. Zhang, N.P. Ong, Z.A. Xu, K. Krishana, R. Gagnon, and L. Taillefer, Determining the Wiedemann-Franz ratio from the thermal Hall conductivity: Application to $\mathrm{Cu}$ and $\mathrm{YBa}_{2} \mathrm{Cu}_{3} \mathrm{O}_{695}$, Phys. Rev. Lett. 84, 2219 (2000), https://doi. org/10.1103/PhysRevLett.84.2219

[9] Y. Wang, L. Li, and N.P. Ong, Nernst effect in high- $T_{c}$ superconductors, Phys. Rev. B 73, 024510 (2006), https://doi.org/10.1103 PhysRevB.73.024510

[10] G. Grissonnanche, A. Legros, S. Badoux, E. Lefrançois, V. Zatko, M. Lizaire, F. Laliberté, A. Gourgout, J.-S. Zhou, S. Pyon, et al., Giant thermal Hall conductivity in the pseudogap phase of cuprate superconductors, Nature 571, 376 (2019), https://doi.org/10.1038/s41586-019-1375-0

[11]J. Chang, N. Doiron-Leyraud, F. Laliberté, R. Daou, D. LeBoeuf, B.J. Ramshaw, R. Liang, D.A. Bonn, W.N. Hardy, C. Proust, et al., Nernst effect in the cuprate superconductor $\mathrm{YBa}_{2} \mathrm{Cu}_{3} \mathrm{O}_{y}$ : Broken rotational and translational symmetries, Phys. Rev. B 84, 014507 (2011), https://doi. org/10.1103/PhysRevB.84.014507

[12] N. Doiron-Leyraud, S. Lepault, O. Cyr-Choinière, B. Vignolle, G. Grissonnanche, F. Laliberté, J. Chang, N. Barišić, M.K. Chan, L. Ji, et al., Hall, Seebeck, and Nernst coefficients of underdoped $\mathrm{HgBa}_{2} \mathrm{CuO}_{4+}$ : Fermi-surface reconstruction in an archetypal cuprate superconductor, Phys. Rev. X 3, 021019 (2013), https://doi.org/10.1103/PhysRevX.3.021019

[13]G. Grissonnanche, F. Laliberté, S. DufourBeauséjour, M. Matusiak, S. Badoux, F.F. Tafti, B. Michon, A. Riopel, O. Cyr-Choinière, J.C. Baglo, et al., Wiedemann-Franz law in the underdoped cuprate superconductor $\mathrm{YBa}_{2} \mathrm{Cu}_{3} \mathrm{O}_{y}$, Phys. Rev. B 93, 064513 (2016), https://doi.org/10.1103 PhysRevB.93.064513

[14]B. Michon, A. Ataei, P. Bourgeois-Hope, C. Collignon, S.Y. Li, S. Badoux, A. Gourgout, F. Laliberté, J.-S. Zhou, N. Doiron-Leyraud, and
L. Taillefer, Wiedemann-Franz law and abrupt change in conductivity across the pseudogap critical point of a cuprate superconductor, Phys. Rev. X 8, 041010 (2018), https://doi.org/10.1103/ PhysRevX.8.041010

[15]M. Matusiak and T. Wolf, Lorenz number in the optimally doped and underdoped superconductor $\mathrm{EuBa}_{2} \mathrm{Cu}_{3} \mathrm{O}_{y}$, Phys. Rev. B 72, 054508(R) (2005), https://doi.org/10.1103/ PhysRevB.72.054508

[16]M. Matusiak, J. Hori, and T. Suzuki, The HallLorenz number in the $\mathrm{La}_{1.855} \mathrm{Sr}_{0.145} \mathrm{CuO}_{4}$ single crystal, Solid State Commun. 139, 376 (2006), https://doi.org/10.1016/j.ssc.2006.06.024

[17]M. Matusiak, K. Rogacki, and B.W. Veal, Enhancement of the Hall-Lorenz number in optimally doped $\mathrm{YBa}_{2} \mathrm{Cu}_{3} \mathrm{O}_{7-\mathrm{d}}$, Euro Phys. Lett. 88, 47005 (2009), https://doi.org/10.1209/0295$5075 / 88 / 47005$

[18] S.I. Mirzaei, D. Stricker, J.N. Hancock, C. Berthod, A. Georges, E. van Heumen, M.K. Chan, X. Zhao, Y. Li, M. Greven, N. Barišić, and D. van der Marel, Spectroscopic evidence for Fermi liquid-like energy and temperature dependence of the relaxation rate in the pseudogap phase of the cuprates, PNAS 110, 5774 (2013), https://doi.org/10.1073/ pnas.1218846110

[19]Y. Li, W. Tabis, G. Yu, N. Barišić, and M. Greven, Hidden Fermi-liquid charge transport in the antiferromagnetic phase of the electron-doped cuprate superconductors, Phys. Rev. Lett. 117, 197001 (2016), https://doi.org/10.1103 PhysRevLett.117.197001

[20]N. Barišić, M.K. Chan, Y. Li, G. Yu, X. Zhao, M. Dressel, A. Smontara, and M. Greven, Universal sheet resistance and revised phase diagram of the cuprate high-temperature superconductors, PNAS 110(30), 12235 (2013), https:// doi.org/10.1073/pnas.1301989110

[21]N. Barišić, M.K. Chan, M.J. Veit, C.J. Dorow, Y. Ge, Y. Li, W. Tabis, Y. Tang, G. Yu, X. Zhao, and M. Greven, Evidence for a universal Fermi-liquid scattering rate throughout the phase diagram of the copper-oxide superconductors, New J. Phys. 21, 113007 (2019), https://doi.org/10.1088/13672630/ab4d0f 
[22]S.-D. Chen, M. Hashimoto, Y. He, D. Song, K.-J. Xu, J.-F. He, T.P. Devereaux, H. Eisaki, D.- H. Lu, J. Zaanen, and Z.-X. Shen, Incoherent strange metal sharply bounded by a critical doping in Bi2212, Science 366, 1099 (2019), https:// doi.org/10.1126/science.aaw8850

[23]J. Zaanen, Planckian dissipation, minimal viscosity and the transport in cuprate strange metals, SciPost Phys. 6, 061 (2019), https://doi. org/10.21468/SciPostPhys.6.5.061

[24]M.V. Sadovskii, Planckian relaxation delusion in metals, Usp. Fiz. Nauk 64(2) (2021), https://doi. org/10.3367/UFNe.2020.08.038821(accepted).

[25]D. Pelc, M.J. Veit, C.J. Dorow, Y. Ge, N. Barišić, and M. Greven, Resistivity phase diagram of cuprates revisited, Phys. Rev. B 102, 075114 (2020), https://doi.org/10.1103/PhysRevB.102.075114

[26]S.A. Hartnoll, Lectures on holographic methods for condensed matter physics, Class. Quant. Grav. 26, 224002 (2009), https://doi.org/10.1088/0264$9381 / 26 / 22 / 224002$

[27]C.P. Herzog, Lectures on holographic superfluidity and superconductivity, J. Phys. A 42, 343001 (2009), https://doi.org/10.1088/1751$8113 / 42 / 34 / 343001$

[28]J. McGreevy, Holographic duality with a view toward many-body physics, Adv. High Energy Phys. 2010, 723105 (2010), https://doi. org/10.1155/2010/723105

[29]S. Sachdev, What can gauge-gravity duality teach us about condensed matter physics? Ann. Rev. Cond. Matt. Phys. 3, 9 (2012), https://doi.org/10.1146/annurev-conmatphys-020911-125141

[30]J. Zaanen, Y. Liu, Y.-W. Sun, and K. Schalm, Holographic Duality in Condensed Matter Physics (Cambridge University Press, 2015), https://doi. org/10.1017/CBO9781139942492

[31]M. Ammon and J. Erdmenger, Gauge/Gravity Duality (Cambridge University Press, 2015), https://doi.org/10.1017/CBO9780511846373

[32]S.A. Hartnoll, A. Lucas, and S. Sachdev, Holographic Quantum Matter (MIT Press, 2018), https://mitpress.mit.edu/books/holographicquantum-matter
[33]R.A. Davison, K. Schalm, and J. Zaanen, Holographic duality and the resistivity of strange metals, Phys. Rev. B 89, 245116 (2014), https://doi. org/10.1103/PhysRevB.89.245116

[34]S.A. Hartnoll and A. Karch, Scaling theory of the cuprate strange metals, Phys. Rev. B 91, 155126 (2015), https://doi.org/10.1103/ PhysRevB.91.155126

[35]J.S. Kim, B.H. Kim, D.C. Kim, and Y.W. Park, Thermoelectric power of $\mathrm{La}_{2-x} \mathrm{Sr}_{x} \mathrm{CuO}_{4}$ at high temperatures, Ann. Phys. 13, 43 (2004), https://doi.org/10.1002/andp.200310041

[36]D.V. Khveshchenko, Viable phenomenologies of the normal state of cuprates, Europhys. Lett. 111, 17003 (2015), https://doi.org/10.1209/02955075/111/17003

[37]G. Aeppli, T.E. Mason, S.M. Hayden, H.A. Mook, and J. Kulda, Nearly singular magnetic fluctuations in the normal state of a high- $T_{c}$ cuprate superconductor, Science 278, 1432 (1997), https://doi.org/10.1126/science.278.5342.1432

[38]R.E. Walstedt, T.E. Mason, G. Aeppli, S.M. Hayden, and H.A. Mook, Pseudogap term in the magnetic response of cuprate superconductors. Phys. Rev. B 84, 024530 (2011), https://doi.org/10.1103/PhysRevB.84.024530

[39]X.-H. Ge, Y. Tian, S.-Y. Wu, and S.-F. Wu, Linear and quadratic in temperature resistivity from holography, JHEP 2016(11), 128 (2016), https://doi.org/10.1007/JHEP11(2016)128

[40]X.-H. Ge, Y. Tian, S.-Y. Wu, and S.-F. Wu, Hyperscaling violating black hole solutions and magneto-thermoelectric DC conductivities in holography, Phys. Rev. D 96, 046015 (2017), https://doi.org/10.1103/PhysRevD.96.046015

[41]S.A. Hartnoll, P.K. Kovtun, M. Müller, and S. Sachdev, Theory of the Nernst effect near quantum phase transitions in condensed matter and in dyonic black holes, Phys. Rev. B 76, 144502 (2007), https://doi.org/10.1103/PhysRevB.76.144502

[42] B.N. Narozhny, I.V. Gornyi, M. Titov, M. Schütt, and A.D. Mirlin, Hydrodynamics in graphene: Linear-response transport, Phys. Rev. B 91, 035414 (2015), https://doi.org/10.1103/ PhysRevB.91.035414 
[43]B.N. Narozhny, I.V. Gornyi, A.D. Mirlin, and J. Schmalian, Hydrodynamic approach to electronic transport in graphene, Ann. Phys. 529, 1700043 (2017), https://doi.org/10.1002 andp.201700043

[44]B.N. Narozhny and M. Schütt, Magnetohydrodynamics in graphene: Shear and Hall viscosities, Phys. Rev. B 100, 5125 (2019), https://doi. org/10.1103/PhysRevB.100.035125

[45]B.N. Narozhny, I.V. Gornyi, and M. Titov, Hydrodynamic collective modes in graphene, arXiv: 2011.03806, https://arxiv.org/abs/2011.03806

[46]D.V. Khveshchenko, Taking a critical look at holographic critical matter, Lith. J. Phys. 55, 208 (2015), https://doi.org/10.3952/physics. v55i3.3150

[47]D.V. Khveshchenko, Demystifying the holographic mystique: A critical review, Lith. J. Phys. 56, 125 (2016), https://doi.org/10.3952/physics. v56i3.3363

[48]B. Goutéraux, Universal scaling properties of extremal cohesive holographic phases, JHEP 1401, 080 (2014), https://doi.org/10.1007 JHEP01(2014)080

[49]B. Goutéraux, Charge transport in holography with momentum dissipation, JHEP 1404, 181 (2014), https://doi.org/10.1007 IHEP04(2014)181

[50]A. Donos and J.P. Gauntlett, Novel metals and insulators from holography, JHEP 2014(06), 007 (2014), https://doi.org/10.1007 JHEP06(2014)007

[51]A. Donos and J.P. Gauntlett, Thermoelectric DC conductivities from black hole horizons, JHEP 2014(11), 081 (2014), https://doi.org/10.1007 JHEP11(2014)081

[52]A. Amoretti, A. Braggio, N. Maggiore, N. Magnoli, and D. Musso, Thermoelectric transport in gauge/gravity models with momentum dissipation, JHEP 2014(09), 160 (2014), https:// doi.org/10.1007/JHEP09(2014)160

[53]A. Amoretti, A. Braggio, N. Magnoli, and D. Musso, Bounds on charge and heat diffusivities in momentum dissipating holography, JHEP 2015(07), 102 (2015), https://doi.org/10.1007 IHEP07(2015)102
[54]A. Amoretti, M. Baggioli, N. Magnoli, and D. Musso, Chasing the cuprates with dilatonic dyons, JHEP 2016(06), 113 (2016), https://doi. org/10.1007/JHEP06(2016)113

[55]A. Amoretti, A. Braggio, N. Maggiore, N. Magnoli, and D. Musso, Analytic dc thermoelectric conductivities in holography with massive gravitons, Phys. Rev. D 91, 025002 (2015), https://doi. org/10.1103/PhysRevD.91.025002

[56]R.A. Davison and B. Goutéraux, Dissecting holographic conductivities, JHEP 2015(09), 090 (2015), https://doi.org/10.1007/JHEP09(2015)090

[57]M. Blake, Momentum relaxation from the fluid/ gravity correspondence, JHEP 2015(09), 010 (2015), https://doi.org/10.1007/JHEP09(2015)010

[58]S.A. Hartnoll and C.P. Herzog, Ohm's law at strong coupling: $S$ duality and the cyclotron resonance, Phys. Rev. D 76, 106012 (2007), https:// doi.org/10.1103/PhysRevD.76.106012

[59]A. Amoretti, D.K. Brattan, N. Magnoli, and M. Scanavino, Magneto-thermal transport implies an incoherent Hall conductivity, JHEP 2020(08), 97 (2020), https://doi.org/10.1007/ JHEP08(2020)097

[60]M. Blake and A. Donos, Quantum critical transport and the Hall angle in holographic models, Phys. Rev. Lett. 114, 021601 (2015), https://doi. org/10.1103/PhysRevLett.114.021601

[61]A. Amoretti and D. Musso, Magneto-transport from momentum dissipating holography, JHEP 2015(09), 094 (2015), https://doi.org/10.1007 JHEP09(2015)094

[62]A. Amoretti, A. Braggio, N. Maggiore, and N. Magnoli, Thermo-electric transport in gauge/ gravity models, Adv. Phys. X 2, 409 (2017), https://doi.org/10.1080/23746149.2017.1300509

[63]S. Cremonini, H.-S. Liu, H. Lü, and C.N. Pope, DC conductivities from non-relativistic scaling geometries with momentum dissipation, JHEP 2017(04), 009 (2017), https://doi.org/10.1007 JHEP04(2017)009

[64]S. Cremonini, A. Hoover, L. Li, and S. Waskie, Anomalous scalings of cuprate strange metals from nonlinear electrodynamics, Phys. Rev. D 99, 061901 (2019), https://doi.org/10.1103 PhysRevD.99.061901 
[65]S. Cremonini, M. Cvetič, and I. Papadimitriou, Thermoelectric DC conductivities in hyperscaling violating Lifshitz theories, JHEP 2018(04), 099 (2018), https://doi.org/10.1007 IHEP04(2018)099

[66]J. Lux and L. Fritz, Interaction-dominated transport and Coulomb drag in bilayer graphene, Phys. Rev. B 87, 075423 (2013), https://doi.org/10.1103/ PhysRevB.87.075423

[67]G. Wagner, D.X. Nguyen, and S.H. Simon, Transport in bilayer graphene near charge neutrality: Which scattering mechanisms are important? Phys. Rev. Lett. 124, 026601 (2020), https:// doi.org/10.1103/PhysRevLett.124.026601

[68]G. Wagner, D.X. Nguyen, and S.H. Simon, Transport properties of multilayer graphene, Phys. Rev. B 101, 245438 (2020), https://doi. org/10.1103/PhysRevB.101.245438

[69]G. Wagner, D.X. Nguyen, and S.H. Simon, Quantum Boltzmann equation for bilayer graphene, Phys. Rev. B 101, 035117 (2020), https://doi.org/10.1103/PhysRevB.101.035117

[70]D.Y.H. Ho, I. Yudhistira, N. Chakraborty, and S. Adam, Theoretical determination of hydrodynamic window in monolayer and bilayer graphene from scattering rates, Phys. Rev. B 97, 121404(R) (2018), https://doi.org/10.1103 PhysRevB.97.121404

[71]C. Tan, D.Y.H. Ho, L. Wang, J.I.A. Li, I. Yudhistira, D.A. Rhodes, T. Taniguchi, K. Watanabe, K. Shepard, P.L. McEuen, et al., Realization of a universal hydrodynamic semiconductor in ultra-clean dual-gated bilayer graphene, arXiv:1908.10921, https://arxiv.org/abs/1908.10921

[72]A. Amoretti, M. Meinero, D.K. Brattan, F. Caglieris, E. Giannini, M. Affronte, C. Hess, B. Buechner, N. Magnoli, and M. Putti, Hydrodynamical description for magneto-transport in the strange metal phase of Bi-2201, Phys. Rev. Res. 2, $023387 \quad$ (2020), https://doi.org/10.1103 PhysRevResearch.2.023387
[73]L.V. Delacrétaz, B. Goutéraux, S.A. Hartnoll, and A. Karlsson, Bad metals from fluctuating density waves, SciPost Phys. 3, 025 (2017), https://doi. org/10.21468/SciPostPhys.3.3.025

[74]L.V. Delacrétaz, B. Goutéraux, S.A. Hartnoll, and A. Karlsson, Bad metals from fluctuating density waves, Phys. Rev. B 96, 195128 (2017), https://doi.org/10.1103/PhysRevB.96.195128

[75] L.V. Delacrétaz, B. Goutéraux, S.A. Hartnoll, and A. Karlsson, Theory of collective magnetophonon resonance and melting of a field-induced Wigner solid, Phys. Rev. B 100, 085140 (2019), https://doi.org/10.1103/PhysRevB.100.085140

[76] A.S. Alexandrov, V.V. Kabanov, and N.F. Mott, Coherent $a b$ and $c$ transport theory of high$T$ cuprates, Phys. Rev. Lett. 77, 4796 (1996), https://doi.org/10.1103/PhysRevLett.77.4796

[77]N. Luo and G.H. Miley, Kohler's rule and relaxation rates in high- $T_{c}$ superconductors, Physica $C$ 371, 259 (2002), https://doi.org/10.1016/S09214534(01)01101-7

[78]D.L. Maslov, V.I. Yudson, and A.V. Chubukov, Resistivity of a non-Galilean-invariant Fermi liquid near Pomeranchuk quantum criticality, Phys. Rev. Lett. 106, 106403 (2011), https://doi. org/10.1103/PhysRevLett.106.106403

[79] H.K. Pal, V.I. Yudson, and D.L. Maslov, Resistivity of non-Galilean-invariant Fermi- and non-Fermi liquids, Lith. J. Phys. 52, 142 (2012), https://doi. org/10.3952/physics.v52i2.2358

[80] M. Swift and C.G. Van de Walle, Conditions for $T^{2}$ resistivity from electron-electron scattering, Eur. Phys. J. B 90, 151 (2017), https://doi.org/10.1140/ epjb/e2017-80367-1

[81]A. Alexandradinata, N.P. Armitage, A. Baydin, W. Bi, Y. Cao, H.J. Changlani, E. Chertkov, E.H. da Silva Neto, L. Delacretaz, I. El Baggari, et al., The future of the correlated electron problem, arXiv:2010.00584, https://arxiv.org abs/2010.00584

\title{
TVARI HOLOGRAFIŠKOJI FENOMENOLOGIJA KUPRATAMS
}

\author{
D.V. Khveshchenko
}

Šiaurès Karolinos universiteto Fizikos ir astronomijos fakultetas, Čepel Hilas, JAV 